\title{
Liver-directed treatments for liver metastasis from gastric adenocarcinoma: comparison between liver resection and radiofrequency ablation
}

\author{
Ali Guner ${ }^{1,2}$ - Taeil Son ${ }^{3,4} \cdot$ In Cho ${ }^{3,5} \cdot$ In Gyu Kwon ${ }^{3,6} \cdot$ Ji Yeong An ${ }^{1,7}$ • \\ Hyoung-Il Kim ${ }^{1,7,8} \cdot$ Jae-Ho Cheong $^{1,7} \cdot$ Sung Hoon Noh ${ }^{1,7} \cdot$ Woo Jin Hyung $^{1,7,8}$ (D)
}

Received: 1 March 2015/Accepted: 19 July 2015/Published online: 1 August 2015

(C) The International Gastric Cancer Association and The Japanese Gastric Cancer Association 2015

\begin{abstract}
Background Although various liver-directed treatment modalities, such as liver resection and radiofrequency ablation (RFA), have been applied to treat liver metastases from gastric cancer, optimal management of them remains controversial. In patients with liver metastasis from gastric cancer, we investigated the short- and long-term outcomes of liver resection and RFA and analyzed factors influencing survival.

Methods A total of 98 gastric cancer patients with liver metastasis and no extrahepatic disease were treated by liver resection $(n=68)$ or RFA $(n=30)$. Short- and long-term
\end{abstract}

A. Guner and T. Son contributed equally to this work.

Woo Jin Hyung

wjhyung@yuhs.ac

1 Department of Surgery, Yonsei University College of Medicine, 50-1 Yonsei-ro, Seodaemun-gu, Seoul 120-752, Republic of Korea

2 Department of General Surgery, Trabzon Kanuni Training and Research Hospital, Trabzon, Turkey

3 Department of Surgery, Graduate School, Yonsei University College of Medicine, Seoul, South Korea

4 Department of Surgery, Eulji Medical Center, Eulji University School of Medicine, Seoul, South Korea

5 Department of Surgery, International St. Mary's Hospital, Incheon, South Korea

6 Department of Surgery, Keimyung University School of Medicine, Daegu, South Korea

7 Gastric Cancer Center, Yonsei Cancer Hospital, Yonsei University Health System, Seoul, South Korea

8 Robot and MIS Center, Severance Hospital, Yonsei University Health System, Seoul, South Korea outcomes were evaluated retrospectively for each of the liver-directed treatments.

Results Severe complication rates did not differ between liver resection $(18 \%)$ and RFA $(10 \%)(p=0.333)$. Only one treatment-related mortality occurred in the liver resection group. No statistically significant difference in survival was noted between the treatment groups. Median overall survival after liver resection was 24 months, with 3-year overall and progression-free survival rates of $40.6 \%$ and $30.4 \%$, respectively. Median overall survival after RFA was 23 months, with 3-year overall and progression-free survival rates of $43.0 \%$ and $37.4 \%$, respectively. Only the size of the metastases was shown to be an independent prognostic factor for gastric cancer patients with liver metastasis.

Conclusions In select patients with liver metastasis from gastric cancer, liver resection and RFA showed satisfactory and comparable short- and long-term results. Thus, systemic chemotherapy may not be the only therapeutic option for patients with liver metastasis, and possible liver-directed treatment options for such patients should be considered on an individual basis.

Keywords Gastric cancer - Liver metastasis - Liver resection - Radiofrequency ablation - Prognosis

\section{Introduction}

Gastric cancer is one of the most common malignancies worldwide and remains the third most common cause of cancer-related death [1]. Although the reported 5-year survival for early gastric cancer is over $90 \%$, patients with advanced disease generally show poor prognosis, even after multimodality treatment, due to distant metastases 
and recurrence $[2,3]$. In gastric cancer, metastasis to the liver is frequent, developing in 5-14\% of all patients with gastric cancer [4-6].

For patients with liver metastasis from gastric cancer, palliative treatment is regarded the standard of care, since their disease is incurable and their life expectancy is dreadfully short. To improve survival, various liver-directed treatment strategies for liver metastasis from gastric cancer, in combination with systemic chemotherapy, have been applied, such as surgical resection, radiofrequency ablation (RFA), and hepatic artery infusion chemotherapy [7-11]. Meanwhile, for liver metastases from colorectal cancer and neuroendocrine tumors, surgical resection is the treatment of choice [12, 13]. RFA, a localized application of thermal energy that induces coagulation necrosis and thereby tumor cell destruction, has also been used to treat primary liver malignancies and liver metastasis from other organ malignancies, including colorectal, breast, and neuroendocrine cancer [14-16].

Nevertheless, although several studies have assessed the safety and efficacy of individual treatment modalities for liver metastases from gastric cancer, few have assessed the outcomes of these treatment modalities in patients with liver metastases from gastric cancer. Therefore, we aimed to investigate the safety and feasibility of liver resection and RFA for liver metastasis from gastric cancer, and to evaluate the oncological outcomes thereof in terms of survival. We also analyzed factors influencing survival in patients who underwent liver-directed treatment.

\section{Methods}

\section{Patients}

From July 1998 to May 2013, a total of 98 gastric cancer patients with liver-only metastasis, either synchronous or metachronous, were treated by liver-directed treatment for metastatic lesions. Among them, surgical resection was performed in 68 patients (liver resection group), while the other 30 patients underwent RFA (RFA group). RFA was introduced in our practice for liver metastasis from gastric cancer in 1998. Accordingly, all patients included in this study had the option of undergoing hepatic resection or RFA as liver-directed treatment modalities. Patient demographics, primary tumor-specific features, metastasisspecific features (size, lobar distribution, number of metastases), and complications based on the ClavienDindo classification system [17] were obtained retrospectively from a review of medical records. Diagnosis of metastasis was based on imaging studies, including computed tomography $(\mathrm{CT})$, magnetic resonance imaging, and positron emission tomography (PET-CT), as well as liver biopsy when possible. Liver metastases were considered synchronous if detected simultaneously or within two months after primary treatment of gastric cancer. This study was approved by the Institutional Review Board of Severance Hospital, Yonsei University Health System (42013-0513).

\section{Treatments}

At our institution, treatment strategies for liver metastases from gastric cancer must be consensually agreed upon by a multidisciplinary team comprising gastric and hepatic surgeons, medical oncologists, and radiologists. Treatment decisions were made on an individual basis, considering the patient's performance status, tumor location, tumor size, and timing of metastasis; decisions were also influenced by the preference of the doctors in charge. All patients included in this study were diagnosed preoperatively using a single or multiple imaging modalities. Also, we performed intraoperative ultrasound for liver resection cases or trans-abdominal ultrasound in cases of percutaneous RFA by a radiologist for all patients included in the study. Thus, we also discovered and treated other lesions that were not detected preoperatively. Accordingly, the number of lesions was counted as that observed on both pre- and intraoperative imaging. In general, multiple liver metastases of bilobar location were not indicated for liverdirected treatment. Also, liver-directed treatments were not indicated if more than four metastatic lesions were present, unless all lesions were unilobar. Meanwhile, liver-directed treatments were commonly indicated in the absence of extrahepatic disease, as defined by nodal metastasis outside of the regional lymph nodes, metastases to other organs, or peritoneal dissemination on preoperative or operative findings. If complete resection of the metastatic lesions was feasible, allowing adequate hepatic reserve, liver resection was preferred. When metastases were smaller than $5 \mathrm{~cm}$ and ineligible for liver resection because of comorbidities and metastasis-specific features, or when patients refused surgery, RFA was considered for treatment of liver metastasis. Since some patients tended to prefer the lessinvasive RFA treatment to the more aggressive resection, they were treated by RFA even when the hepatic lesion was resectable.

All patients underwent liver-directed treatment for liver metastasis with curative intent. For patients with synchronous lesions, gastrectomy with systemic lymphadenectomy was also performed. All patients with metachronous lesions in this study had previously undergone radical gastrectomy with systemic lymphadenectomy. Both anatomic and nonanatomic resections-including wedge resection and hemihepatectomy-were performed, depending on the number, size, and locations of the 
metastatic lesions. For RFA, a single $17-\mathrm{G}$ cooled-tip electrode (Cool-tip RF ablation system, Valleylab, Boulder, CO, USA) was used for $12 \mathrm{~min}$ under real-time ultrasonographic guidance. The number of ablations was determined based on tumor size, and a single puncture, double puncture, or overlapping technique was used. After the electrode was connected to the generator, radiofrequency energy was emitted to each tumor site using an impedance control algorithm. All RFA procedures were performed by radiologists for both intraoperative and percutaneous application. RFA treatment responses were evaluated by contrast-enhanced computed tomography at four weeks after the procedure. After liver-directed treatment, chemotherapy was recommended to all patients regardless of whether they received liver resection or RFA. Early in the study period, anthracycline- and cisplatinbased chemotherapeutic regimens were commonly used. More recently, however, TS-1 plus cisplatin or capecitabine plus oxaliplatin for 6-12 months, based on the performance status of the patient, was more commonly used.

\section{Follow-up}

Mortality was defined as death occurring within 30 days of treatment. Progression or recurrence was documented with various imaging studies. Patients with progression or recurrence were classified as locoregional-only, liver-only, or liver recurrence with other systemic metastases or systemic metastases without liver recurrence. In this study, we only defined the location where progression or recurrence appeared first.

\section{Statistical analysis}

All statistical analyses were performed using statistical analysis software (SPSS 20.0, IBM Corp., Armonk, NY, USA). The distribution of continuous data was tested using the Kolmogorov-Smirnov test. Data were analyzed using the $\chi^{2}$ test, Mann-Whitney test, or Student's $t$ test, depending on whether the variable was categorical or continuous. Overall survival was calculated from the date of liver-directed treatment to either the date of death from any cause or the last follow-up date. Progression-free survival was defined as the time from liver-directed treatment to first documentation of recurrence or progression. During the study period, patients were followed from the date of liverdirected treatment until June 30, 2014 or their death. The median follow-up period was 24 months (range 4-189) for all patients; that of the liver resection group was 25 months, while that of the RFA group was 23 months. The follow-up period for survivors until the last follow-up date ranged from 13 to 189 months, with a median of 63 months. The Kaplan-Meier method was used to determine survival, and the log rank test was used to compare groups. A forward stepwise procedure in Cox proportional hazards regression analysis was used to conduct multivariate analysis in order to discover whether the type of liver-directed treatment could serve as a prognostic factor for overall survival or progression-free survival. For all statistical tests, $p<0.05$ was considered statistically significant.

\section{Results}

\section{Patient characteristics}

Among the 68 patients in the liver resection group, 26 patients $(38 \%)$ were diagnosed with synchronous metastasis, while 42 patients $(62 \%)$ had metachronous metastasis. In the RFA group, 13 of 30 patients (43\%) presented with synchronous metastasis, while the remaining 17 (57\%) exhibited metachronous metastasis. In the RFA group, liver metastases were diagnosed in 8 patients $(27 \%)$ by a single modality, such as CT or PET-CT alone, and by multiple modalities, including liver biopsy, in 22 patients (73\%), compared to 28 patients $(41 \%)$ and 40 patients $(59 \%)$, respectively, in the liver resection group $(p=0.162)$. There was no difference in the pathological characteristics of primary gastric lesions between the liver resection and RFA groups. The clinicopathologic characteristics of the liver metastatic lesions, including number, size, and lobar distribution, were also similar between the two treatment groups. Chemotherapy was recommended to all patients in both groups; however, four patients $(4 \%)$ did not receive chemotherapy because they declined or because of in-hospital mortality (Table 1).

For liver resection, 23 patients (34\%) underwent wedge resection, $24(35 \%)$ underwent segmentectomy, and 21 patients $(31 \%)$ underwent hemihepatectomy. In the RFA group, 14 patients $(47 \%)$ were treated intraoperatively via laparotomy, and 16 patients (53\%) underwent RFA via a percutaneous approach.

\section{Morbidity and mortality}

In the liver resection group, 19 patients $(28 \%)$ had complications, including $12(18 \%)$ severe complications (grade III or higher by Clavien-Dindo classification). Seven patients $(23 \%)$ in the RFA group experienced posttreatment complications, including three $(10 \%)$ severe complications. Only one $(1.5 \%)$ treatment-related mortality was noted: the patient underwent a right-inferior segmentectomy and died on postoperative day 19 due to hepatic insufficiency. There was no statistically significant difference in either morbidity or mortality between the two treatment groups (Table 2). 
Table 1 Clinicopathologic characteristics of all treated patients

\begin{tabular}{|c|c|c|c|}
\hline & \multicolumn{2}{|c|}{ Number of patients } & \multirow[t]{2}{*}{$p$ value } \\
\hline & $\operatorname{RFA}(n=30)$ & Liver resection $(n=68)$ & \\
\hline Age $(\text { years })^{\mathrm{a}}$ & $60(31-84)$ & $61(30-75)$ & 0.456 \\
\hline Gender & & & 0.307 \\
\hline Male & $22(73 \%)$ & $56(82 \%)$ & \\
\hline Female & $8(27 \%)$ & $12(18 \%)$ & \\
\hline ASA class & & & 0.229 \\
\hline ASA I & $12(40 \%)$ & $33(48 \%)$ & \\
\hline ASA II & $14(47 \%)$ & $33(48 \%)$ & \\
\hline ASA III & $4(13 \%)$ & $2(4 \%)$ & \\
\hline Tumor location & & & 0.472 \\
\hline Upper & $4(13 \%)$ & $12(18 \%)$ & \\
\hline Mid & $6(20 \%)$ & $14(20 \%)$ & \\
\hline Lower & $19(64 \%)$ & $42(62 \%)$ & \\
\hline Mixed & $1(3 \%)$ & - & \\
\hline Extent of gastrectomy & & & 0.433 \\
\hline Subtotal & $21(70 \%)$ & $42(62 \%)$ & \\
\hline Total & $9(30 \%)$ & $26(38 \%)$ & \\
\hline $\mathrm{T}$ classification ${ }^{\mathrm{b}}$ & & & 0.961 \\
\hline $\mathrm{T} 1$ & $3(10 \%)$ & $10(15 \%)$ & \\
\hline $\mathrm{T} 2$ & $6(20 \%)$ & $7(10 \%)$ & \\
\hline $\mathrm{T} 3$ & $8(27 \%)$ & $23(34 \%)$ & \\
\hline $\mathrm{T} 4 \mathrm{a}$ & $13(43 \%)$ & $28(41 \%)$ & \\
\hline $\mathrm{N}$ classification ${ }^{\mathrm{b}}$ & & & 0.051 \\
\hline No & $4(13 \%)$ & $17(25 \%)$ & \\
\hline N1 & $5(17 \%)$ & $15(22 \%)$ & \\
\hline $\mathrm{N} 2$ & $5(17 \%)$ & $14(21 \%)$ & \\
\hline N3 & $16(53 \%)$ & $22(32 \%)$ & \\
\hline Number of retrieved lymph nodes ${ }^{\mathrm{a}}$ & $38(10-86)$ & $40(8-85)$ & 0.990 \\
\hline Number of metastatic lymph nodes ${ }^{\mathrm{a}}$ & $7(0-31)$ & $3(0-45)$ & 0.082 \\
\hline Pathological stage ${ }^{\mathrm{b}}$ & & & 0.496 \\
\hline Stage I & $4(13 \%)$ & $10(15 \%)$ & \\
\hline Stage II & $1(3 \%)$ & $14(21 \%)$ & \\
\hline Stage III & $14(47 \%)$ & $18(26 \%)$ & \\
\hline Stage IV & $11(37 \%)$ & $26(38 \%)$ & \\
\hline Histology ${ }^{c}$ & & & 0.962 \\
\hline Differentiated & $20(67 \%)$ & $45(66 \%)$ & \\
\hline Undifferentiated & $10(33 \%)$ & $23(34 \%)$ & \\
\hline Lymphatic invasion & & & 0.860 \\
\hline Yes & $16(53 \%)$ & $35(52 \%)$ & \\
\hline No & $9(30 \%)$ & $18(26 \%)$ & \\
\hline Unknown & $5(17 \%)$ & $15(22 \%)$ & \\
\hline Venous invasion & & & 0.245 \\
\hline Yes & $13(43 \%)$ & $36(53 \%)$ & \\
\hline No & $11(37 \%)$ & $17(25 \%)$ & \\
\hline Unknown & $6(20 \%)$ & $15(22 \%)$ & \\
\hline Perineural invasion & & & 0.283 \\
\hline Yes & $4(13 \%)$ & $19(28 \%)$ & \\
\hline No & $11(37 \%)$ & $26(38 \%)$ & \\
\hline Unknown & $15(50 \%)$ & $23(34 \%)$ & \\
\hline
\end{tabular}


Table 1 continued

\begin{tabular}{|c|c|c|c|}
\hline & \multicolumn{2}{|c|}{ Number of patients } & \multirow[t]{2}{*}{$p$ value } \\
\hline & $\operatorname{RFA}(n=30)$ & Liver resection $(n=68)$ & \\
\hline \multicolumn{3}{|l|}{ Lauren class } & \multirow[t]{5}{*}{0.628} \\
\hline Intestinal & $17(57 \%)$ & $38(56 \%)$ & \\
\hline Diffuse & $3(10 \%)$ & $7(10 \%)$ & \\
\hline Mixed & $3(10 \%)$ & $3(4 \%)$ & \\
\hline Not available & $7(23 \%)$ & $20(30 \%)$ & \\
\hline Number of metastases ${ }^{a}$ & $1(1-4)$ & $1(1-6)$ & \multirow[t]{4}{*}{0.498} \\
\hline 1 & $22(73 \%)$ & $45(66 \%)$ & \\
\hline 2 & $5(17 \%)$ & $15(22 \%)$ & \\
\hline$\geq 3$ & $3(10 \%)$ & $8(12 \%)$ & \\
\hline Size of metastases ${ }^{\mathrm{a}}(\mathrm{cm})$ & $2.2(0.5-5.8)$ & $2.7(0.6-10)$ & 0.087 \\
\hline \multicolumn{3}{|l|}{ Lobar distribution } & \multirow[t]{3}{*}{0.283} \\
\hline Unilobar & $24(80 \%)$ & $60(88 \%)$ & \\
\hline Bilobar & $6(20 \%)$ & $8(12 \%)$ & \\
\hline \multicolumn{3}{|l|}{ Timing of metastasis } & \multirow[t]{3}{*}{0.635} \\
\hline Synchronous & $13(43 \%)$ & $26(38 \%)$ & \\
\hline Metachronous & $17(57 \%)$ & $42(62 \%)$ & \\
\hline \multicolumn{3}{|l|}{ Diagnostic modalities } & \multirow[t]{6}{*}{0.162} \\
\hline CT alone & $4(13 \%)$ & $15(22 \%)$ & \\
\hline PET-CT & $4(13 \%)$ & $13(19 \%)$ & \\
\hline $\mathrm{CT}+\mathrm{MRI}$ & $11(37 \%)$ & $26(38 \%)$ & \\
\hline $\mathrm{MRI}+\mathrm{PET}-\mathrm{CT}$ & $9(30 \%)$ & $14(21 \%)$ & \\
\hline Liver biopsy after $\mathrm{CT} \pm$ MRI & $2(7 \%)$ & - & \\
\hline \multicolumn{3}{|l|}{ Adjuvant chemotherapy } & \multirow[t]{3}{*}{0.390} \\
\hline Yes & $28(93 \%)$ & $66(97 \%)$ & \\
\hline No & $2(7 \%)$ & $2(3 \%)$ & \\
\hline \multicolumn{4}{|c|}{$\begin{array}{l}R F A \text { radiofrequency ablation, } A S A \text { American Society of Anesthesiologists, } C T \text { computed tomography, } M R I \\
\text { magnetic resonance imaging, } P E T-C T \text { positron emission tomography }\end{array}$} \\
\hline \multicolumn{4}{|c|}{${ }^{a}$ Presented as median value (range) } \\
\hline \multicolumn{4}{|c|}{$\begin{array}{l}\text { b Stage of gastric cancer was determined according to the 7th American Joint Committee on Cancer } \\
\text { guidelines }\end{array}$} \\
\hline
\end{tabular}

\section{Survival and recurrence}

The median overall survival for the liver resection group was 24 months, with 1, 3, and 5-year overall survivals of $79.1,40.6$, and $30.0 \%$, respectively. In the RFA group, the 1,3 , and 5-year overall survivals of patients were 73.3, 43.0 , and $34.4 \%$, respectively, with a median survival of 23 months. There were no statistically significant differences in overall survival between the treatment groups, regardless of the timing of metastasis $(p=0.807$, $p=0.606$, and $p=0.844$; for all patients, synchronous metastasis, and metachronous metastasis, respectively; Fig. 1).
After excluding the mortality case, in the follow-up period, 39 patients $(40.2 \%)$ showed no progression or recurrence. The other 58 patients $(59.8 \%)$ exhibited progressions in the liver only (46.5\%), in the liver and other organs $(41.4 \%)$, and in organs other than the liver $(12.1 \%)$. The types of progression according to treatment and timing of metastasis are presented in Fig. 2.

In the liver resection group, the 1-, 3-, and 5-year progression-free survivals were $49.3,30.4$, and $26.0 \%$, respectively. In the RFA group, the 1-, 3-, and 5-year progression-free survivals were $56.7,37.4$, and $32.8 \%$, respectively. There was no statistically significant difference in progression-free survival between the treatment 
Table 2 Comparison of early outcomes for liver-directed treatments

\begin{tabular}{llll}
\hline & RFA $(n=30)$ & Liver resection $(n=68)$ & $p$ value \\
\hline Overall complications & $7(23 \%)$ & $19(28 \%)$ & 0.634 \\
Severe complications & $3(10 \%)$ & $12(18 \%)$ & 0.333 \\
Liver abscess $^{\mathrm{a}}$ & $2(7 \%)$ & - & \\
Hepatic insufficiency $^{\mathrm{a}}$ & - & $1(2 \%)$ & \\
Biloma $^{\mathrm{a}}$ & - & $3(4 \%)$ & \\
Intra-abdominal bleeding $^{\mathrm{b}}$ & $1(3 \%)$ & - & \\
Intra-abdominal abscess $^{\mathrm{b}}$ & - & $4(6 \%)$ & \\
Intra-abdominal fluid collection $^{\mathrm{b}}$ & - & $3(4 \%)$ & \\
Anastomosis leakage & - & $1(2 \%)$ & 0.504 \\
Mortality & - & $1(1.5 \%)$ &
\end{tabular}

RFA radiofrequency ablation

${ }^{a}$ Liver-directed treatment-specific complications

b Gastrectomy-specific complications groups for all treated patients, patients with synchronous metastases, and patients with metachronous metastases $(p=0.790, \quad p=0.558$, and $p=0.519$, respectively; Fig. 1).

In the multivariate analysis of overall survival for all treated patients, larger size of liver metastasis $(\geq 3 \mathrm{~cm})$ [hazard ratio (HR), 1.905; $p=0.016$ ] was shown to be independently associated with poor overall survival. While no independent prognostic factors were noted for synchronous metastasis, larger-size metastases $(\geq 3 \mathrm{~cm})$ (HR, 2.803; $p=0.005$ ) and sex (female) (HR, 2.605; $p=0.020$ ) were shown to be independent factors of poor prognosis for metachronous metastasis (Table 3). Results from multivariate analysis of progression-free survival were similar to those for overall survival. For all treated patients and for synchronous metastasis, no prognostic factors were discovered. Meanwhile, as was the case in overall survival, larger-size metastases $(\geq 3 \mathrm{~cm}$ ) (HR, 2.163; $p=0.025$ ) was shown to be an independent factor of poor prognosis for metachronous metastasis (Table 4).

\section{Discussion}

In the present study, RFA and liver resection for patients with liver metastasis from gastric cancer demonstrated acceptable morbidity and mortality. Both treatments were feasible not only for metachronous metastasis but also for synchronous metastasis with simultaneous gastrectomy and systemic lymphadenectomy. Moreover, RFA and liver resection showed comparable and favorable long-term outcomes, with median survivals of almost 2 years in gastric cancer patients with either synchronous or metachronous liver metastases, although patients were highly selected.
Fig. 1 Kaplan-Meier plots of estimated overall survival and progression-free survival from the date of liver-directed treatment: a overall survival curves for all treated patients; b progression-free survival curves for all treated patients; $\mathbf{c}$ overall survival for patients with synchronous metastasis; $\mathbf{d}$ progression-free survival for patients with synchronous metastasis; e overall survival for patients with metachronous metastasis; $\mathbf{f}$ progression-free survival for patients with metachronous metastasis. $R F A$ radiofrequency ablation

Following recent advances in treatment options for malignant diseases, prognoses after treatment of metastatic gastrointestinal malignancies have been improved, particularly for liver metastases from colorectal and neuroendocrine cancer [16, 18, 19]. However, managing liver metastasis from gastric cancer remains a challenge; gastric cancer patients with liver metastasis demonstrate a median survival of less than 1 year with chemotherapy alone [20]. To improve survival therein, liver-directed treatment modalities have been introduced, although highly varying survival rates have been reported [21-24]. In the present study, despite their technical limitations, RFA and liver resection demonstrated comparable and favorable shortterm and long-term outcomes that suggest the possibility of applying these modalities in select patients. To achieve better prognoses, local control of metastases is crucial. Therefore, current treatment modalities for liver metastasis focus on local disease control [25]. Nevertheless, the liver remains the dominant site for progression after liver-directed treatments [26], and our findings confirmed that any recurrence or progression would likely involve the liver in the majority of patients. However, despite the high rate of liver involvement in progressed patients, $40.2 \%$ of all patients experienced no progression or recurrence during the follow-up period. Thus, although the treatment groups consisted of highly selected patients, the improved progression-free outcomes demonstrated in the present study 

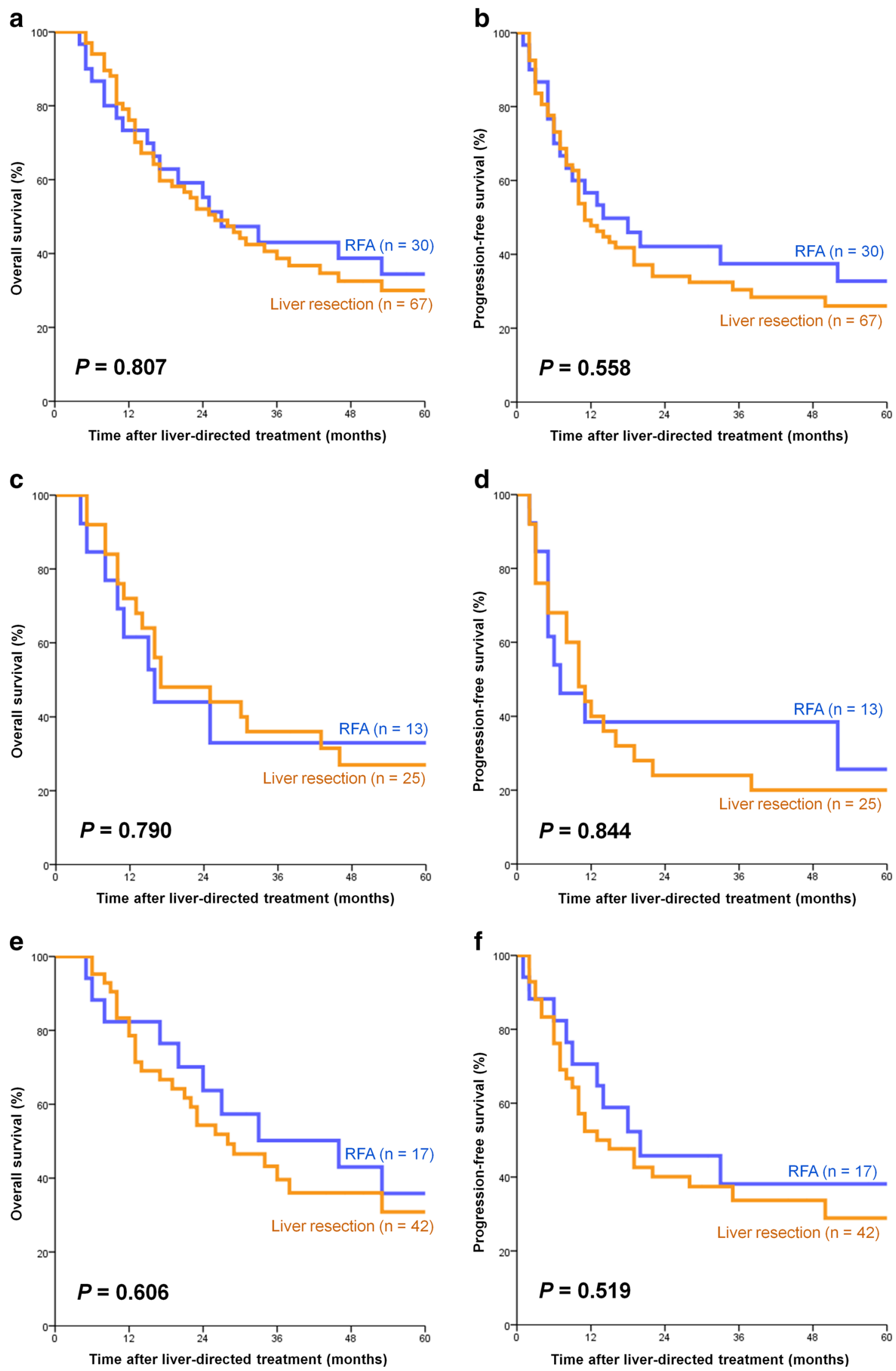


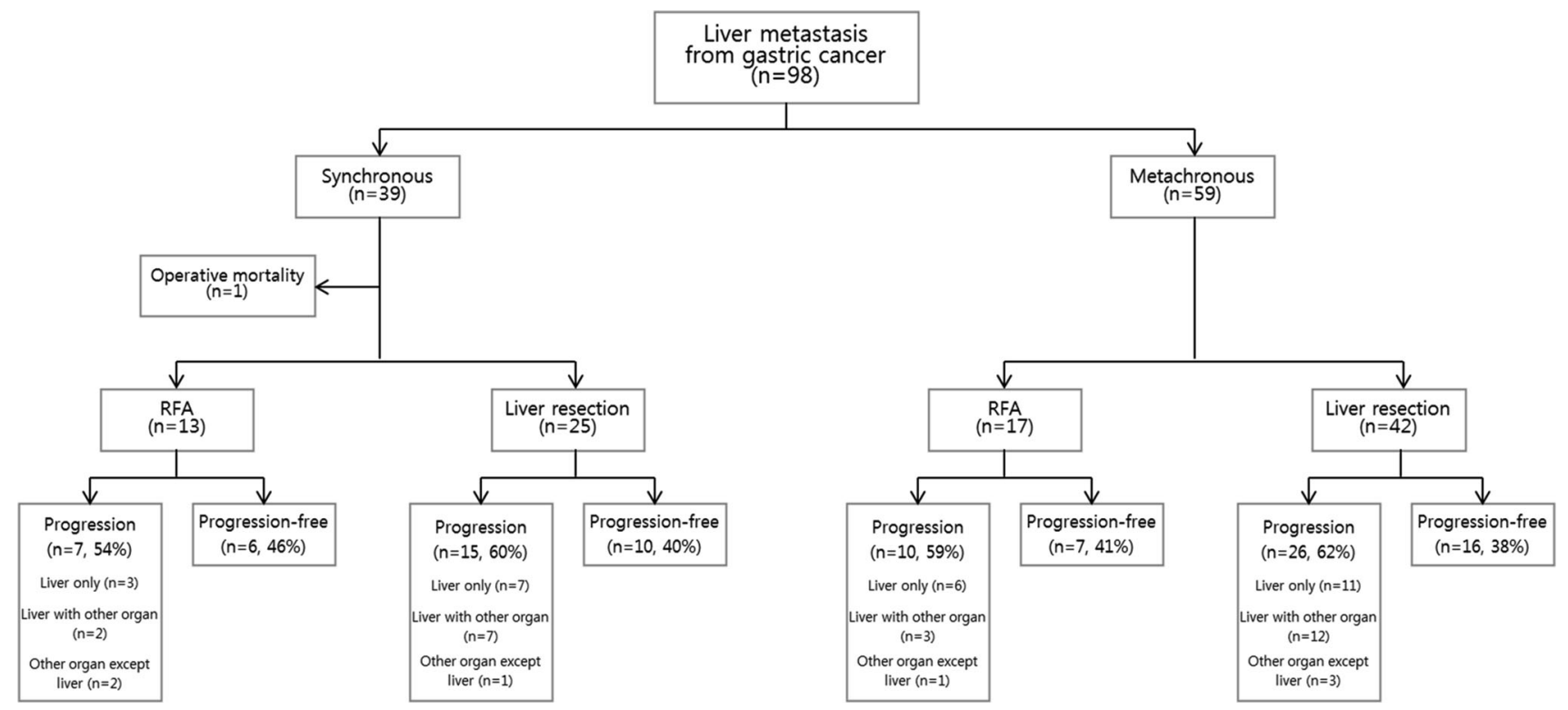

Fig. 2 Progression patterns in patients from both treatment groups according to the timing of metastasis. $R F A$ radiofrequency ablation

Table 3 Multivariate analysis of overall survival

\begin{tabular}{|c|c|c|c|c|c|c|}
\hline & \multicolumn{2}{|l|}{ All patients } & \multicolumn{2}{|c|}{ Synchronous metastasis } & \multicolumn{2}{|c|}{ Metachronous metastasis } \\
\hline & Hazard ratio & $p$ value & Hazard ratio & $p$ value & Hazard ratio & $p$ value \\
\hline Treatment (RFA vs liver resection) & $\begin{array}{l}1.113 \\
\quad(0.648-1.910)\end{array}$ & 0.698 & $\begin{array}{l}0.900(0.406- \\
1.994)\end{array}$ & 0.794 & $\begin{array}{l}1.218 \\
(0.581-2.551)\end{array}$ & 0.601 \\
\hline \multicolumn{7}{|l|}{ Age $(\leq 60$ vs $>60)$} \\
\hline Gender (male vs female) & & & & & $\begin{array}{l}2.605 \\
\quad(1.165-5.824)\end{array}$ & 0.020 \\
\hline \multicolumn{7}{|l|}{ Extent of gastrectomy (subtotal vs total) } \\
\hline \multicolumn{7}{|l|}{ Depth of invasion (serosa negative vs positive) } \\
\hline \multicolumn{7}{|l|}{ Lymph node metastasis (absent vs present) } \\
\hline \multicolumn{7}{|l|}{ Histology (differentiated vs undifferentiated) } \\
\hline \multicolumn{7}{|l|}{ Number of metastases (single vs multiple) } \\
\hline Size of metastases $(<3 \mathrm{vs} \geq 3 \mathrm{~cm})$ & $\begin{array}{l}1.905 \\
\quad(1.126-3.223)\end{array}$ & 0.016 & & & $\begin{array}{l}2.803 \\
\quad(1.361-5.774)\end{array}$ & 0.005 \\
\hline \multicolumn{7}{|l|}{ Lobar distribution (unilobar vs bilobar) } \\
\hline Timing (synchronous vs metachronous) & & & - & & - & \\
\hline
\end{tabular}

suggest that both treatments could provide local control for stage IV disease when limited to the liver.

Proper patient selection is key to the success of liverdirected treatment. Although the application of liver-directed treatments for liver metastasis from gastric cancer is increasing, the exact indications for their use have not been defined. In fact, liver-directed treatments are rarely indicated, and patient groups that are indicated for such treatments comprise a very small fraction of individuals with hepatic metastasis from gastric cancer due to the multisystemic metastatic nature of gastric cancer, as reflected in data previously reported by our institution $[4,22]$. Therefore, when deciding on a particular management strategy, investigators must consider various selection criteria, such as unilobar distribution, solitary tumors, and absence of extrahepatic disease [21]. Among these, absence of extrahepatic metastasis seems the most reasonable. In the present study, we only provided liver-directed treatment for patients without extrahepatic disease. For patients with liver-only metastasis, metastasis-specific features were evaluated individually to determine the feasibility of RFA or resection [27, 28]. Meanwhile, various prognostic factors, such as solitary lesion or synchronous presentation, have also been previously suggested to improve patient selection $[10,26]$. In the present study, metastasis size was the only metastasis-specific feature that was shown to be 
Table 4 Multivariate analysis of progression-free survival

\begin{tabular}{|c|c|c|c|c|c|c|}
\hline & \multicolumn{2}{|l|}{ All patients } & \multicolumn{2}{|c|}{ Synchronous metastasis } & \multicolumn{2}{|c|}{ Metachronous metastasis } \\
\hline & Hazard ratio & $p$ value & Hazard ratio & $p$ value & Hazard ratio & $p$ value \\
\hline Treatment (RFA vs liver resection) & $\begin{array}{l}1.168 \\
\quad(0.686-1.988)\end{array}$ & 0.567 & $\begin{array}{l}1.079 \\
(0.491-2.375)\end{array}$ & 0.849 & $\begin{array}{l}1.120 \\
\quad(0.538-2.334)\end{array}$ & 0.762 \\
\hline \multicolumn{7}{|l|}{ Age $(\leq 60$ vs $>60)$} \\
\hline \multicolumn{7}{|l|}{ Gender (male vs female) } \\
\hline \multicolumn{7}{|l|}{ Extent of gastrectomy (subtotal vs total) } \\
\hline \multicolumn{7}{|c|}{ Depth of invasion (serosa negative vs positive) } \\
\hline \multicolumn{7}{|l|}{ Lymph node metastasis (absent vs present) } \\
\hline \multicolumn{7}{|l|}{ Histology (differentiated vs undifferentiated) } \\
\hline \multicolumn{7}{|l|}{ Number of metastases (single vs multiple) } \\
\hline Size of metastases $(<3 \mathrm{vs} \geq 3 \mathrm{~cm})$ & & & & & $\begin{array}{l}2.163 \\
\quad(1.103-4.239)\end{array}$ & 0.025 \\
\hline \multicolumn{7}{|l|}{ Lobar distribution (unilobar vs bilobar) } \\
\hline Timing (synchronous vs metachronous) & & & - & - & - & - \\
\hline
\end{tabular}

Values in parentheses are $95 \%$ confidence intervals

$R F A$ radiofrequency ablation

independently associated with prognosis in all treated patients. Nevertheless, only patients suitable for liver-directed treatment were selected for inclusion in the present study, so the characteristics of the liver metastases were relatively favorable: the mean size of the metastatic lesions was $2.6 \mathrm{~cm}$, around $70 \%$ were single lesions, and $85 \%$ were unilobar in distribution. However, because of the lack of consensus on patient selection criteria, useful indicators for properly selecting patients for individual treatment modalities are yet to be established.

Along with the selection of appropriate patients, choice of treatment modality is just as important. Various factors, such as timing of metastasis, metastatic features, and technical limitations of a procedure, should be considered when deciding on a particular treatment $[29,30]$. Additionally, a patient's performance status should also be taken into consideration when selecting a surgical or nonsurgical modality. In the present study, liver resection was preferred for larger metastases or metastases that were easy to resect due to location, regardless of timing of metastasis. RFA was chosen in cases where resection would have been difficult or the patient refused surgery. Due to the similarities in short- and long-term outcomes, we believe that both modalities should be considered to be complementary to each other, and one should be selected over the other after considering their technical limitations and patient factors.

A few limitations warrant consideration when interpreting the results of this study. One limitation is the retrospective nature of the study. Nevertheless, it would be somewhat difficult to obtain a large enough number of eligible patients to conduct a prospective study. To address this problem, a multicenter study with well-defined inclusion criteria, including different modalities, is warranted. As well, a randomized study comparing different modalities may provide stronger evidence regarding the validity of liver-directed treatment. However, all modalities have their own technical limitations, such as the feasibility of resection for multiple metastases and the reduced efficacy of RFA for tumors of large size or those close to the major vessels; thus, it would be difficult to ensure homogeneous treatment groups. The varying indications created by technical limitations of the procedures might have induced selection bias. However, the baseline clinicopathologic characteristics of the patients in both treatment groups in this study were comparable. Also, all patients had undergone at least D2 lymphadenectomy, and most of the patients received post-treatment chemotherapy. Diagnostic confirmation of metastasis is another problem for patients undergoing liver-directed treatment. In the present study, we mostly used more than one modality, including liver biopsy, to reach a diagnosis in order to overcome this concern. Recent advances in imaging modalities with which to diagnose hepatic lesions may help to increase the sensitivity and specificity in diagnosing hepatic metastasis [31]. Notwithstanding, there would still remain the small possibility of treating primary liver tumors in RFA groups.

In conclusion, RFA and liver resection provide comparable short-term and long-term outcomes. Both can be applied as safe and effective modalities for treating liver metastasis from gastric cancer, if patients are selected properly. In selecting an appropriate patient and treatment modality, surgeons should individually consider the characteristics of the patient and their liver metastasis. We 
believe that liver-directed treatment options could offer gastric cancer patients with liver metastasis improved survival. Thus, systemic chemotherapy may not be the only therapeutic option for all patients with liver metastasis, and possible liver- directed treatment options for such patients should be considered on an individual basis.

Acknowledgments This study was supported by Yonsei University College of Medicine [grant number: 6-2007-0097]. The authors would like to thank Anthony Thomas Milliken, ELS (Editing Synthase, Seoul, Korea) for his help with the editing of this manuscript.

\section{Compliance with ethical standards}

Conflict of interest The authors declare no conflicts of interest.

\section{References}

1. GLOBOCAN 2012. Estimated cancer incidence, mortality and prevelance worldwide in 2012. Lyon: International Agency for Research on Cancer. http://www.globocan.iarc.fr.

2. Lai JF, Kim S, Kim K, et al. Prediction of recurrence of early gastric cancer after curative resection. Ann Surg Oncol. 2009;16:1896-902.

3. Yoo CH, Noh SH, Shin DW, Choi SH, Min JS. Recurrence following curative resection for gastric carcinoma. $\mathrm{Br} \mathrm{J}$ Surg. 2000;87:236-42.

4. Cheon SH, Rha SY, Jeung HC, et al. Survival benefit of combined curative resection of the stomach (D2 resection) and liver in gastric cancer patients with liver metastases. Ann Oncol. 2008;19:1146-53.

5. Sakamoto Y, Sano T, Shimada K, et al. Favorable indications for hepatectomy in patients with liver metastasis from gastric cancer. J Surg Oncol. 2007;95:534-9.

6. Okano K, Maeba T, Ishimura K, et al. Hepatic resection for metastatic tumors from gastric cancer. Ann Surg. 2002;235:86-91.

7. Kakeji Y, Morita M, Maehara Y. Strategies for treating liver metastasis from gastric cancer. Surg Today. 2010;40:287-94.

8. Kodera Y, Fujitani K, Fukushima N, et al. Surgical resection of hepatic metastasis from gastric cancer: a review and new recommendation in the Japanese gastric cancer treatment guidelines. Gastric Cancer. 2014;17:206-12.

9. Ojima H, Ootake S, Yokobori T, et al. Treatment of multiple liver metastasis from gastric carcinoma. World J Surg Oncol. 2007;5:70.

10. Garancini M, Uggeri F, Degrate L, et al. Surgical treatment of liver metastases of gastric cancer: is local treatment in a systemic disease worthwhile? HPB (Oxford). 2012;14:209-15.

11. Yamakado K, Nakatsuka A, Takaki H, et al. Prospective study of arterial infusion chemotherapy followed by radiofrequency ablation for the treatment of liver metastasis of gastric cancer. J Vasc Interv Radiol. 2005;16:1747-51.

12. Morris EJ, Forman D, Thomas JD, et al. Surgical management and outcomes of colorectal cancer liver metastases. Br J Surg. 2010;97:1110-8.
13. Page AJ, Weiss MJ, Pawlik TM. Surgical management of noncolorectal cancer liver metastases. Cancer. 2014;120:3111-21.

14. Bortolotto C, Macchi S, Veronese L, Dore R, Draghi F, Rossi S. Radiofrequency ablation of metastatic lesions from breast cancer. J Ultrasound. 2012;15:199-205.

15. Liu Y, Li S, Wan X, et al. Efficacy and safety of thermal ablation in patients with liver metastases. Eur J Gastroenterol Hepatol. 2013;25:442-6.

16. Mazzaglia PJ, Berber E, Milas M, Siperstein AE. Laparoscopic radiofrequency ablation of neuroendocrine liver metastases: a 10 -year experience evaluating predictors of survival. Surgery. 2007;142:10-9.

17. Dindo D, Demartines N, Clavien P-A. Classification of surgical complications. Ann Surg. 2004;240:205-13.

18. Oshowo A, Gillams A, Harrison E, Lees WR, Taylor I. Comparison of resection and radiofrequency ablation for treatment of solitary colorectal liver metastases. Br J Surg. 2003;90:1240-3.

19. Reddy SK, Barbas AS, Marroquin CE, Morse MA, Kuo PC, Clary BM. Resection of noncolorectal nonneuroendocrine liver metastases: a comparative analysis. J Am Coll Surg. 2007;04:372-82.

20. Glimelius B, Ekstrom K, Hoffman K, et al. Randomized comparison between chemotherapy plus best supportive care with best supportive care in advanced gastric cancer. Ann Oncol. 1997;8:163-8.

21. Makino H, Kunisaki C, Izumisawa Y, et al. Indication for hepatic resection in the treatment of liver metastasis from gastric cancer. Anticancer Res. 2010;30:2367-76.

22. Kim HR, Cheon SH, Lee KH, et al. Efficacy and feasibility of radiofrequency ablation for liver metastases from gastric adenocarcinoma. Int J Hyperthermia. 2010;26:305-15.

23. Baek HU, Kim SB, Cho EH, et al. Hepatic resection for hepatic metastases from gastric adenocarcinoma. J Gastric Cancer. 2013;13:86-92.

24. Kim HO, Hwang SI, Hong HP, Yoo CH. Radiofrequency ablation for metachronous hepatic metastases from gastric cancer. Surg Laparosc Endosc Percutan Tech. 2009;19:208-12.

25. Minami Y, Kudo M. Radiofrequency ablation of liver metastases from colorectal cancer: a literature review. Gut Liver. 2013;7:1-6.

26. Kerkar SP, Kemp CD, Avital I. Liver resections in metastatic gastric cancer. HPB (Oxford). 2010;12:589-96.

27. Koga R, Yamamoto J, Ohyama S, et al. Liver resection for metastatic gastric cancer: experience with 42 patients including eight long-term survivors. Jpn J Clin Oncol. 2007;37:836-42.

28. Chen J, Tang Z, Dong X, et al. Radiofrequency ablation for liver metastasis from gastric cancer. Eur J Surg Oncol. 2013;39:701-6.

29. Mulier S, Ni Y, Jamart J, Ruers T, Marchal G, Michel L. Local recurrence after hepatic radiofrequency coagulation. Ann Surg. $2005 ; 242: 158-71$.

30. Thelen A, Jonas S, Benckert C, et al. Liver resection for metastatic gastric cancer. Eur J Surg Oncol. 2008;34:1328-34.

31. Kim YK, Lee MW, Lee WJ, et al. Diagnostic accuracy and sensitivity of diffusion-weighted and of gadoxetic acid-enhanced 3-T MR imaging alone or in combination in the detection of small liver metastasis $(\leq 1.5 \mathrm{~cm}$ in diameter). Invest Radiol. 2012;47:159-66. 